\title{
Injection of hTERT-Transduced Endothelial Progenitor Cells Promotes Beneficial Aortic Changes in a High-Fat Dietary Model of Early Atherosclerosis
}

\author{
Xingsheng $\mathrm{Li}^{\mathrm{a}}$ Yanru Deng $^{\mathrm{b}}$ Shunrong Zhao ${ }^{\mathrm{a}}$ Dandan Zhang ${ }^{\mathrm{a}}$ \\ Qingwei Chen ${ }^{\text {a }}$ \\ a Department of Gerontology, The Second Affiliated Hospital of Chongqing Medical University, Chongqing, and \\ ${ }^{\mathrm{b}}$ Intensive Care Unit, Anhui Provincial Hospital Affiliated to Anhui Medical University, Hefei, China
}

\section{Key Words}

Endothelium · Endothelial progenitor cells · Human

telomerase catalytic subunit $\cdot$ Atherosclerosis

\begin{abstract}
Objectives: Cultured endothelial progenitor cells (EPCs) display troubling issues that adversely affect their applicability to endothelial regeneration. We hypothesized that transduction of the human telomerase catalytic subunit (hTERT) gene would enhance EPC function in treating dietary-induced early atherosclerosis (AS). Methods: A dietary-induced early AS model was successfully constructed in 90 healthy male rats, while 30 healthy control $(\mathrm{HC})$ rats were normally fed. Four experimental groups were constructed: an untreated $\mathrm{HC}$ group; an untreated AS group injected with PBS; a null EPC AS group injected with null vector-transduced EPCs, and an hTERT EPC AS group injected with hTERT-transduced EPCs. Two months postinjection, abdominal aortas were extracted to validate EPC integration and comparatively assess mRNA and protein expression of the early atherosclerotic markers VCAM-1, ICAM-1, LFA-1, Mac-1, CD44, MCP-1, endothelial nitric oxide synthase (eNOS), and apolipoprotein E. Results: In
\end{abstract}

\section{KARGER}

(c) 2016 S. Karger AG, Basel

E-Mail karger@karger.com

www.karger.com/crd vitro, hTERT transduction of EPCs resulted in a significantly superior proliferative capacity as well as significantly higher NO, iNOS, and LDH secretory capacity. In vivo injection of hTERT-transduced EPCs produced significant reductions in CD44 and MCP-1 expression as well as a significant increase in eNOS expression relative to injection with null vectortransduced EPCs (all $p<0.05$ ). Conclusion: hTERT-transduced human EPCs may be useful in treating dietary-induced early AS.

(c) 2016 S. Karger AG, Basel

\section{Introduction}

Damage to the endothelial monolayer has been shown to be a key milestone in the development of early atherosclerotic lesions as well as the progression of plaque formation [1]. For instance, high-resolution tomography has demonstrated that endothelial dysfunction is positively associated with infiltration of inflammatory macrophages and microchannel development [2]. Moreover, accelerated development of atherosclerotic lesions has been observed in vessel areas with lower levels of endo- 
thelial-protective molecules [3]. Accordingly, improving endothelial function has been linked to a lower risk of adverse cardiovascular events [1].

On the basis of this evidence, the maintenance of a healthy endothelium can be critical to the prevention and treatment of early atherosclerotic disease [4]. To proactively address this challenge, the endothelial monolayer can be regenerated through the injection of endothelial progenitor cells (EPCs) into the general circulation, which can forestall the development of atherosclerotic lesions [4]. Although this technique shows promise, cultured EPCs display troubling issues with respect to proliferation, apoptosis, and karyotypic change, which adverselyaffect their applicability to endothelial regeneration [5]. In order to address these issues, there has been increasing interest in promoting telomerase activity in cultured EPCs in order to improve their applicability to endothelial regeneration [5].

In this study, we hypothesized that transduction of the human telomerase catalytic subunit (hTERT) gene would enhance EPC function in treating dietary-induced early AS. Specifically, an exogenous hTERT gene was transduced into cultured rat EPCs in order to establish a stable in vitro population of hTERT+ EPCs. These hTERT+ EPCs were then injected into a high-fat dietary rat model of early AS in order to assess their effects in treating dietary-induced early AS. As early atherosclerotic lesion formation involves the migration of activated monocytes/ macrophages into the subendothelial space of the arterial wall, we specifically assessed changes in key adhesion markers, including vascular cell adhesion molecule-1 (VCAM-1), intercellular adhesion molecule-1 (ICAM-1), the leukocytic ICAM-1 ligands lymphocyte function-associated antigen-1 (LFA-1; CD11a/CD18) and macrophage-1 antigen (Mac-1; CD11b/CD18), the adhesion molecule CD44, and the monocytic chemokine MCP-1 [6]. We also assessed changes in the tonal regulator endothelial nitric oxide synthase (eNOS) and apolipoprotein E (apoE) [6]. This study should aid our understanding of the effects of cultured EPCs upon early atherosclerotic plaque formation as well as future development of an hTERT-transduced human EPC line for treating dietaryinduced AS.

\section{Materials and Methods}

Ethics Statement

The experimental protocols involving animal subjects conformed to the Guide for the Care and Use of Laboratory Animals (National Institutes of Health, Bethesda, Md., USA) and were ap- proved by the Institutional Animal Care and Use Committee of the Second Affiliated Hospital of Chongqing Medical University (Chongqing, China).

\section{EPC Extraction}

As previously described by Wang et al. [7] with minor modifications, a combination of femoral, humeral, and tibial bone marrow were extracted from healthy male Sprague-Dawley rats (200$300 \mathrm{~g}$ ) by flushing the bone marrow with cold physiological saline. Bone marrow mononuclear cells were then isolated with Histopaque-1083.25 through density-gradient centrifugation. They were then resuspended in EGM-2 (Hyclone, Tianjin, China) for incubation at $37^{\circ} \mathrm{C}$ and $5 \% \mathrm{CO}_{2}$ for 15 days in vitro (DIV), with the medium being replaced every 3 days in vitro.

\section{Quantitative FACS of EPCs}

After 15 days in vitro and immediately prior to EPC injection, quantitative FACS (fluorescence-activated cell sorting) of EPCs was performed as previously described by Kalka et al. [8] with minor modifications. Briefly, cells were gently detached using trypsin and PBS with $1 \mathrm{mM}$ EDTA. Then, the detached cells $\left(\sim 2 \times 10^{5}\right.$ cells $)$ were incubated with the following monoclonal antibodies at $4^{\circ} \mathrm{C}$ for 30 min: anti-kinase insert domain-containing receptor (KDR; Sigma, Beijing, China), anti-vascular endothelium (VE)-cadherin (Chemicon, Temecula, Calif., USA), FITC-conjugated anti-CD3 (Becton Dickinson, San Jose, Calif., USA), phycoerythrin-conjugated anti-CD14, phycoerythrin-conjugated anti-CD19 (Becton Dickinson), phycoerythrin-conjugated anti-CD31 (Becton Dickinson), phycoerythrin-conjugated anti-CD34 (Becton Dickinson), FITC-conjugated anti-CD51/61 (Pharmingen, San Diego, Calif., USA), and FITC-conjugated anti-CD68 (Dako, Carpinteria, Calif., USA). Isotype-identical antibodies were used as controls (Pharmingen). In order to perform the anti-KDR and anti-VE-cadherin analysis, cells were also incubated with FITC-conjugated antistreptavidin (Caltag, South San Francisco, Calif., USA) and biotinylated anti-IgG $(\mathrm{H}+\mathrm{L}$; Vector Laboratories, Burlingame, Calif., USA). Following antibody treatment, the stained cells were fixed with $1 \%$ paraformaldehyde. A Becton Dickinson FACStar flow cytometer was used to perform the quantitative FACS. Cell number versus log (fluorescence intensity) histograms were prepared for $\sim 2 \times 10^{4}$ cells/sample.

Construction of Recombinant hTERT Adeno-Associated Viral Vector (rAAV-GFP-hTERT)

Vector construction was performed as previously described by Wang et al. [7] with minor modifications. Briefly, total RNA was extracted from fetal rat liver tissue and stored at $-80^{\circ} \mathrm{C}$, and then $10 \mu \mathrm{g}$ of fetal rat RNA was used to synthesize single-stranded cDNA. Rat hTERT was PCR amplified from the cDNA with the following primer sequences: sense primer $5^{\prime}$-CCC GGA CCT CCA TCA GAG C- $3^{\prime}$ and antisense primer $5^{\prime}$-CAC CGG CCT TAT TCC AAG C-3'. PCR was performed with two units of LA Taq polymerase (TaKaRa Biotechnology, Dalian, China) as follows in a 50 - $\mu$ volume: $4 \mathrm{~min}$ at $94^{\circ} \mathrm{C}$, and then 35 cycles of $1 \mathrm{~min}$ at $94^{\circ} \mathrm{C}$, $45 \mathrm{~s}$ at $52^{\circ} \mathrm{C}$, and then $1 \mathrm{~min}$ at $72^{\circ} \mathrm{C}$, and then $10 \mathrm{~min}$ at $72^{\circ} \mathrm{C}$. The resulting PCR product was extracted, digested with restriction enzymes, and recloned into the pAAV-IRES-GFP expression plasmid (Stratagene, Shanghai, China) under CMV promoter control. The resulting rAAV-hTERT-IRES-GFP vector (hereinafter $\mathrm{rAAV}$ hTERT-GFP) was analyzed through digestion with restriction en- 
zymes. Vector construction was validated through sequencing by the Shanghai Invitrogen Biological Technology Co. Ltd. (Shanghai, China).

AAV-2 vector amplification was performed as previously described by Wang et al. [7] using the AAV Helper-Free System (Stratagene). Briefly, 100 plates of HEK293 cells (100 $\mathrm{cm}^{2}$ plates) were cultured to $\sim 80 \%$ confluence. Using a standardized calcium phosphate-based procedure, the HEK293 cells were cotransduced with the recombinant plasmid or the control plasmid (pAAV-hrGFP), pHelper, and pAAVRC. Twenty-four hours after transduction, the HEK293 cells were placed in new Dulbecco's minimum essential medium with $10 \%$ newborn calf serum. Sixty hours after transduction, rAAV were released from the HEK293 cells through repeated freeze-thaw cycling. Single-step column purification of AAV2 through gravity-driven flow was used to purify the rAAV vectors based on heparin affinity. Dot blotting was used to measure viral titers. Sodium dodecyl sulfate polyacrylamide gel electrophoresis (SDS-PAGE) was used to assess the purity of rAAV-hTERT-GFP.

Vector Transduction and Validation of hTERT Expression

Vector transduction into cultured EPCs and validation of hTERT expression through reverse transcription PCR (RT-PCR) were performed as previously described by Wang et al. [7] with minor modifications. After the cultured EPCs achieved an $80 \%$ confluence, the EPCs were transduced with either the rAAV-GFP null vector or the rAAV-hTERT-GFP vector $\left(1 \times 10^{4}\right.$ to $5 \times 10^{5} \mathrm{vg}$ per cell) in EGM-2 at $37^{\circ} \mathrm{C}$ for $2 \mathrm{~h}$. After the 2-hour transduction period, the old EGM-2 was replaced with fresh EGM-2 containing $10 \%$ FBS. After $72 \mathrm{~h}$, transgene expression was validated by inverted fluorescent microscopy (data not shown).

At $48 \mathrm{~h}$ following fluorescent microscopic validation, total RNA was extracted using Trizol reagent (Invitrogen). Then, AMV reverse transcriptase (TaKaRa Biotechnology) was used to reverse transcribe the cDNA for PCR amplification. The following rat hTERT primer sequences were used for PCR amplification: forward primer $5^{\prime}$-GTA TGC CGT GGT CCA GAA GG-3' and reverse primer 5 '-CGT GGG TGA GGT GAG GTG TC- 3 '. The following primers were used for the $\beta$-actin control: forward primer of $5^{\prime}$-CGT TGA CAT CCG TAA AGA- $3^{\prime}$ and reverse primer $5^{\prime}$ AGC CAC CAA TCC ACA CAG-3'. As a result, hTERT mRNA expression was validated (data not shown).

\section{EPC Proliferation Assay}

In order to verify and compare the proliferative capacity of transduced EPCs, we performed a proliferation assay as previously described by Li et al. [5]. The three groups of cells (nontransduced control EPCs, rAAV-GFP null vector-transduced EPCs, and rAAV-hTERT-GFP-transduced EPCs) were plated in parallel onto 96-well plates, and proliferation was assayed for 1-7 days in vitro. At the end of each 24-hour measurement period, MTT (5 $\mathrm{mg} / \mathrm{ml}$, Sigma) was added to the culture medium for $4 \mathrm{~h}$ at $37^{\circ} \mathrm{C}$ and $5 \% \mathrm{CO}_{2}$. After removal of the cell culture, $150 \mu$ of dimethyl sulfoxide was applied to the cells followed by $10 \mathrm{~min}$ on a lowspeed oscillator in a dark room. The optical density of each well was assessed at a 492-nm wavelength by a microplate reader. Experiments were performed in triplicate on each individual sample.

EPC Assays for NO, iNOS, and $L D H$

In order to verify and compare the secretory capacity of transduced EPCs, we performed assays for NO, iNOS, and LDH as pre- viously described by Li et al. [5]. The three groups of cells (nontransduced control EPCs, rAAV-GFP null vector-transduced EPCs, and rAAV-hTERT-GFP-transduced EPCs) were plated in parallel onto 96-well plates. Secreted NO concentrations - based on measuring spectrophotometrically determined nitrite concentrations with Griess reagents using a microplate reader (Bio-Rad, Hercules, Calif., USA) at $540 \mathrm{~nm}$ - were assessed with a commercial assay kit according to the manufacturer's instructions (Nanjing Jiancheng Bioengineering Institute, Nanjing, China). iNOS activity levels - based on measuring iNOS-specific NO generation by sodium nitroprusside - were assessed with a commercial assay kit according to the manufacturer's instructions (Nanjing Jiancheng Bioengineering Institute). Secreted LDH concentrations - based on measuring spectrophotometrically determined $\mathrm{LDH}$ concentrations with a microplate reader (Bio-Rad) at 340 $\mathrm{nm}$ - were assessed with a commercial assay kit according to the manufacturer's instructions (Beyotime Institute of Biotechnology, Haimen, Jiangsu, China). Experiments were performed in triplicate on each individual sample.

\section{Construction of the Rat Model of Early AS and EPC Injection}

The rat model of dietary-induced early AS (the AS model) was constructed as previously described by Wang et al. [7] with minor modifications. A total of 120 healthy male Sprague-Dawley rats (200-300 g) were randomly segregated into 30 healthy control (HC) rats and 90 AS rats. The $\mathrm{HC}$ rats were fed a normal diet for 3 months, while the AS rats were fed a high-fat diet. After 3 months of dietary treatment, ultrasonography was able to validate the successful construction of the AS model through comparatively assessing aortic changes between the $\mathrm{HC}$ and AS rats (data not shown). Moreover, serum total cholesterol, low-density lipoprotein cholesterol (LDL-C), triglyceride, and glucose levels were assayed with a biochemical analyzer (Unicel DxC 800, Beckman Coulter, Fullerton, Calif., USA) in order to verify that all AS rats showed statistically similar levels prior to EPC injection.

In order to construct the experimental groups, the rats were injected (via the tail vein) as follows: the HC group was not injected; the untreated AS group was injected with $1 \mathrm{ml}$ of PBS; the null EPC AS group was injected with rAAV-GFP-transduced EPCs $\left(2 \times 10^{6}\right.$ cells suspended in $1 \mathrm{ml}$ of PBS at $72 \mathrm{~h}$ posttransduction), and the hTERT EPC AS group was injected with rAAV-hTERTGFP-transduced EPCs $\left(2 \times 10^{6}\right.$ cells suspended in $1 \mathrm{ml}$ of PBS at $72 \mathrm{~h}$ posttransduction). All subjects survived the experimental protocol and were fed a normal diet postinjection.

\section{Postinjection Aortic Tissue Collection and Preparation}

As previously described by Wang et al. [7] with minor modifications, all rats were euthanized via intravenous $\mathrm{KCl}$ at 2 months postinjection. The abdominal aorta was dissected out and rinsed 10 times with ice-cold PBS. The aorta was immediately placed in liquid nitrogen and then frozen down at $-80^{\circ} \mathrm{C}$ for later analysis.

\section{Validation of EPC Integration by Inverted Fluorescent}

Microscopy

In order to validate the incorporation of GFP+ EPCs into the recipient aortic endothelium, EPC integration into the collected aortic tissue samples was assessed as previously described by Wang et al. [7] with minor modifications. The tissue portion kept at $-80^{\circ} \mathrm{C}$ was placed into an optimal-cutting temperature compound at $-20^{\circ} \mathrm{C}$. With a cryostat, $10-\mu \mathrm{m}$ sections were prepared 
for glass slides and then imaged via inverted fluorescent microscopy. Experiments were performed in triplicate on each individual sample.

\section{Validation of Aortic Tissue hTERT Expression by} Immunoblotting

hTERT expression in the aortic tissue samples was assessed as previously described by Wang et al. [7] with minor modifications. Immunoblotting was applied to validate hTERT protein expression in the aortic samples. Total protein was extracted from each aortic sample, separated by $10 \%$ SDS-PAGE, and finally transferred to a nitrocellulose membrane. A polyclonal rabbit anti-rat hTERT antibody (diluted 1:400; Santa Cruz Biotechnology, Santa Cruz, Calif., USA) and a horseradish peroxidase-conjugated secondary antibody (diluted 1:2,000; Santa Cruz Biotechnology) were then applied. $\beta$-Actin (diluted 1:400; Santa Cruz Biotechnology) was employed as the control. An ECL Plus kit (GE Healthcare Life Science, Piscataway, N.J., USA) was used to assess the hTERT signal intensity, which confirmed positive hTERT protein expression in the hTERT EPC AS group (data not shown). Experiments were performed in triplicate on each individual sample.

\section{mRNA Expression Analysis of Early AS Markers}

As previously described by Wang et al. [7] with minor modifications, mRNA expression of VCAM-1, ICAM-1, LFA-1, Mac-1, CD44, MCP-1, eNOS, and apoE in the collected aortic tissue samples were assessed via RT-PCR. Briefly, total RNA was extracted using Trizol reagent (Invitrogen), and AMV reverse transcriptase (TaKaRa Biotechnology) was used to reverse transcribe cDNA for PCR amplification. The following primer sequences were used for PCR amplification: VCAM-1 forward primer $5^{\prime}$-GAG ACA AAA CAG AAG TGG AAT- $3^{\prime}$ and VCAM-1 reverse primer $5^{\prime}$-AGC AAC GTT GAC ATA AAG AGT-3'; ICAM-1 forward primer 5' TAT CGG GAT GGT GAA GTC T- $3^{\prime}$ and ICAM-1 reverse primer $5^{\prime}$-GGC GGT AAT AGG TGT AAA TG-3'; LFA-1 forward primer $5^{\prime}$-GTC TCA GAG CTA CAG GGT GAC C- $3^{\prime}$ and LFA-1 reverse primer $5^{\prime}$-CAC CTC GTG CAC TGG GAT TTC- ${ }^{\prime}$; Mac1 forward primer $5^{\prime}$-GAT GCT TAC TTG GGT TAT GCT T- ${ }^{\prime}$ and Mac- 1 reverse primer $5^{\prime}$-CGA GGT GCC CCT AAA ACC A-3'; CD44 forward primer $5^{\prime}$-GCA CAC CTA CCT TCC TAC-3' and CD44 reverse primer $5^{\prime}$-CTG GAA TCT GAG GTC TCC TC3'; MCP-1 forward primer 5'-TCA GCC AGA TGC AGT TAA CGC- $3^{\prime}$ and $\mathrm{MCP}-1$ reverse primer $5^{\prime}$-TGA TCC TCT TGT AGC TCT CCA GC- $3^{\prime}$; eNOS forward primer $5^{\prime}$-CAC CGA TAC AAC ATA CTT GAG G- $3^{\prime}$ and eNOS reverse primer $5^{\prime}$-CAG AGC CAT ACA GGA TAG TCG-3'; apoE forward primer $5^{\prime}$-GAC GGA AGT AAA GGC ATA CAA A- $3^{\prime}$ and apoE reverse primer $5^{\prime}$-GCT CAC GGA TAG CAC TCA CA- $3^{\prime}$; and $\beta$-actin forward primer $5^{\prime}$ CGT TGA CAT CCG TAA AGA- $3^{\prime}$ and $\beta$-actin reverse primer $5^{\prime}$-AGC CAC CAA TCC ACA CAG-3'. Data were calculated using the $2^{-\Delta \Delta C t}$ Pfaffl method [9], where the Ct values of the three experimental groups (untreated AS group, null EPC AS group, and hTERT EPC AS group) were compared to those of the HC group and normalized to the $\mathrm{Ct}$ values of $\beta$-actin. Experiments were performed in triplicate on each individual sample.

\section{Biotinylation and Immunoblotting Analysis of Cell Surface} Markers

Expression of the cell surface markers VCAM-1, ICAM-1, LFA-1, Mac-1, and CD44 in the collected aortic tissue samples

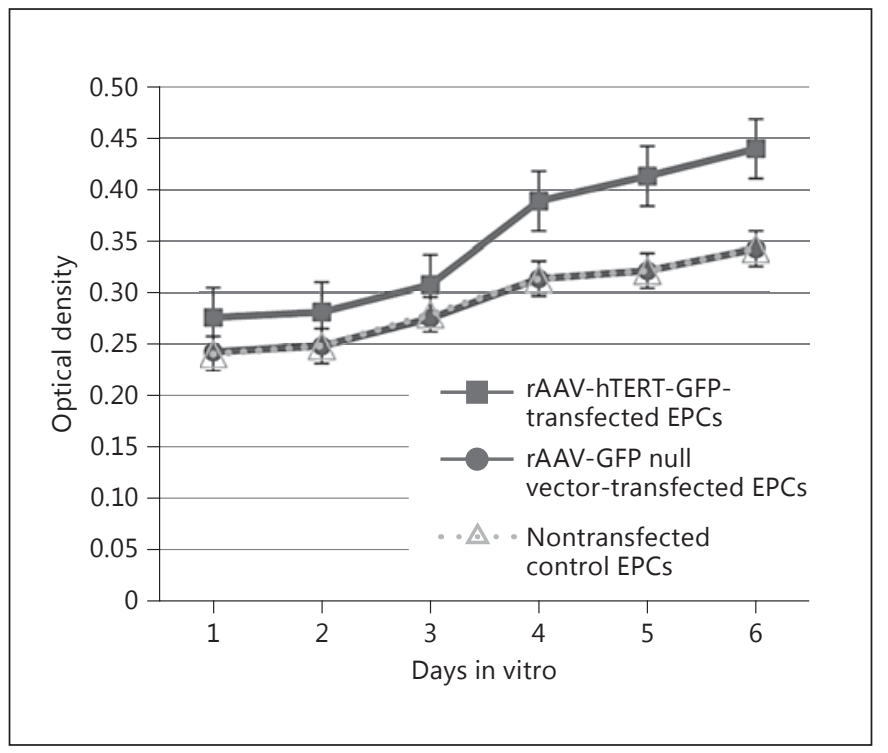

Fig. 1. EPC proliferation assay. A proliferation assay was performed to verify and compare the proliferative capacity of the three groups of EPCs: nontransduced control EPCs, rAAV-GFP null vector-transduced EPCs, and rAAV-hTERT-GFP-transduced EPCs. hTERT-transduced EPCs displayed a significantly greater proliferative capacity relative to the null vector-transduced EPCs and nontransduced EPCs $(\mathrm{p}<0.05)$. Null vector-transduced EPCs and nontransduced EPCs displayed nearly identical proliferation rates $(\mathrm{p}>0.05)$.

were assessed by biotinylation and immunoblotting analysis as previously described by Lei et al. [10] with minor modifications. Briefly, aortic samples were labeled with EZ-link sulfo-NHS-SSbiotin $(0.5 \mathrm{mg} / \mathrm{ml}$; Pierce Biotechnology, Rockford, Ill., USA) for 30 min on ice. After washing the samples thrice with ice-cold PBS to remove the excess biotin, $80 \mu \mathrm{l}$ of extraction buffer was added to each aortic sample. Cells were lightly scrapped off, and the cell suspension was transferred to a tube for $15 \mathrm{~min}$ of centrifugation at $13,000 \mathrm{~g}$ at $4^{\circ} \mathrm{C}$. The resulting supernatant from each sample was treated with $30 \mu \mathrm{l}$ of neutravidin beads (Pierce Biotechnology) for $4 \mathrm{~h}$. The beads were then washed with $1 \mathrm{ml}$ of extraction buffer four times and resuspended in $1 \times$ SDS sample buffer. Protein extraction, SDS-PAGE, and immunoblotting were performed as described above. Anti-Na+/K+ ATPase al (1:200; Alomone Labs, Jerusalem, Israel) was used as the positive control, while anti- $\beta$ galactosidase (1:5,000; Novus, Littleton, Colo., USA) was used as the negative control.

\section{Statistical Analysis}

SPSS version 19.0 (SPSS Inc., Chicago, Ill., USA) and Microsoft Excel 2013 (Microsoft, Redmond, Wash., USA) were used to conduct all statistical analyses. All data are reported as means with standard deviations from triplicate experiments where noted. Comparisons between groups were performed with Student's $\mathrm{t}$ test and nonparametric Kruskal-Wallis one-way analysis of variance. A p value $<0.05$ was deemed to be of statistical significance. 


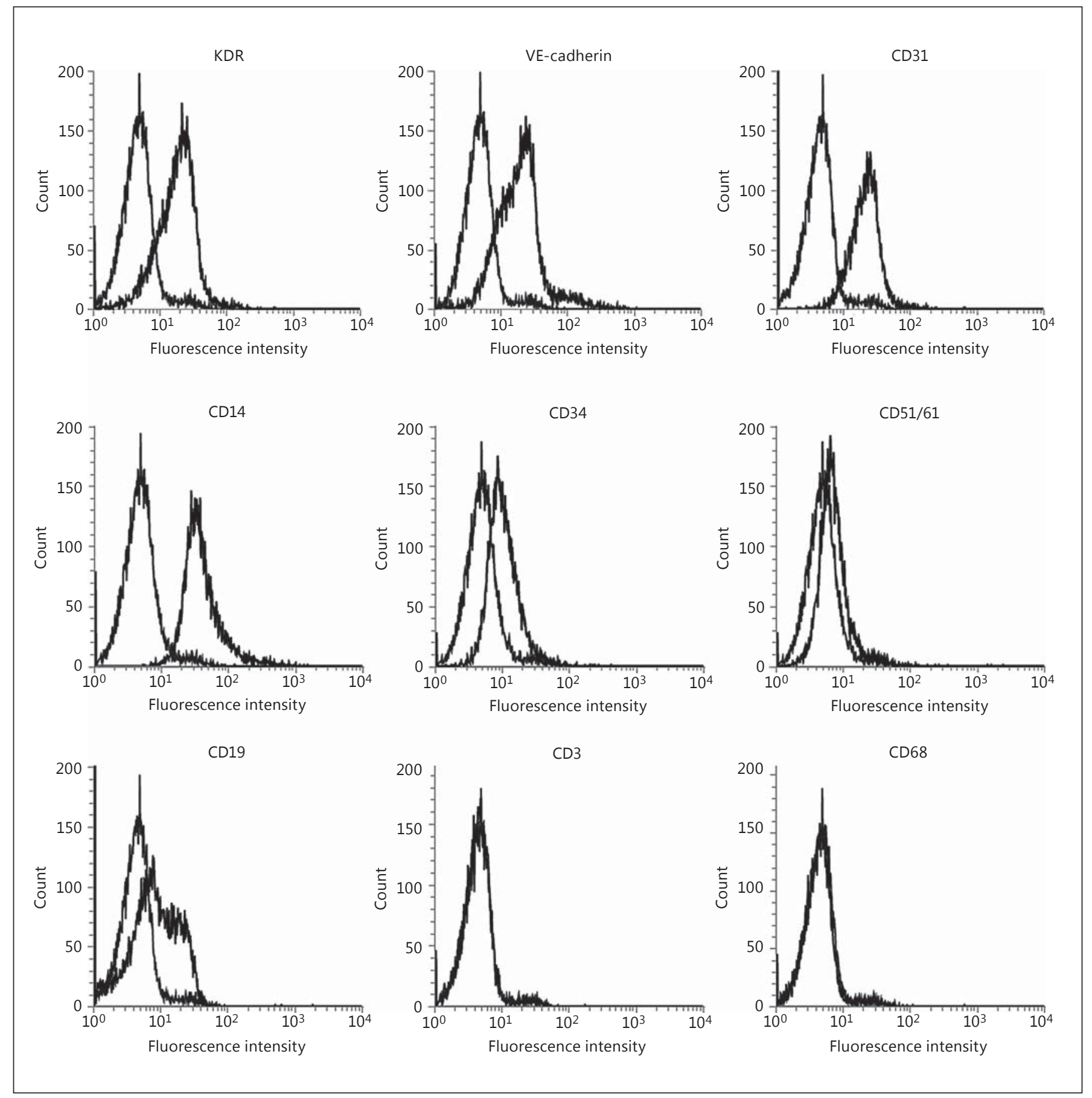

Fig. 2. Quantitative FACS of EPCs. Representative quantitative FACS of cell surface markers immediately prior to in vivo injection. Staining histograms of the five key endothelial surface markers - KDR, VE-cadherin, CD31, CD14, and CD34 - are displayed, showing significantly increased expression relative to their respective isotype controls. The nonendothelial surface markers -
CD51/61, CD19, CD3, and CD68 - did not show significantly increased expression relative to their respective isotype controls. The fluorescence intensity ( $\mathrm{x}$-axis) is proportionate to the antigen's surface abundance, while the corresponding cell count is displayed on the y-axis. 
Table 1. Concentration levels of NO, iNOS, and LDH secreted from cultured EPCs

\begin{tabular}{llll}
\hline Group & NO, $\mu \mathrm{M}$ & iNOS, U/ml & LDH, $\mu \mathrm{M}$ \\
\hline Control EPCs & $14.74 \pm 0.44$ & $4.03 \pm 0.05$ & $3.54 \pm 0.12$ \\
Null EPCs & $14.85 \pm 0.92$ & $3.31 \pm 0.23^{*}$ & $3.53 \pm 0.04$ \\
hTERT EPCs & $17.63 \pm 0.23^{*}$ & $4.28 \pm 0.29^{*}$ & $4.08 \pm 0.04^{*}$ \\
\hline
\end{tabular}

$* \mathrm{p}<0.05$ compared to the EPC group.

\begin{tabular}{lllll}
\hline Group & $\begin{array}{l}\text { Total cholesterol, } \\
\mathrm{mM}\end{array}$ & $\begin{array}{l}\text { LDL-C, } \\
\mathrm{mM}\end{array}$ & $\begin{array}{l}\text { Triglycerides, } \\
\mathrm{mM}\end{array}$ & $\begin{array}{l}\text { Glucose, } \\
\mathrm{mM}\end{array}$ \\
\hline HC & $1.09 \pm 0.01$ & $0.71 \pm 0.19$ & $1.05 \pm 0.58$ & $3.59 \pm 2.15$ \\
Untreated AS & $2.27 \pm 0.41^{*}$ & $1.22 \pm 0.12^{*}$ & $2.02 \pm 0.56^{*}$ & $\begin{array}{l}7.29 \pm 1.97^{*} \\
\text { Null EPC AS }\end{array}$ \\
$\begin{array}{l}2.29 \pm 0.20^{*} \\
\text { hTERT EPC AS }\end{array}$ & $2.27 \pm 0.08^{*}$ & $1.08 \pm 0.18^{*}$ & $1.82 \pm 0.54^{*}$ & $6.68 \pm 1.84^{*}$ \\
\hline
\end{tabular}

$* \mathrm{p}<0.05$ compared to the HC group.
Table 2. Serum levels of total cholesterol, LDL-C, triglycerides, and glucose across the groups prior to EPC injection for NO, iNOS, and LDH on the three groups of cultured EPCs: nontransduced control EPCs, rAAV-GFP null vector-transduced EPCs, and rAAV-hTERT-GFP-transduced EPCs. As a result, hTERT-transduced EPCs displayed significantly greater $\mathrm{NO}$, iNOS, and $\mathrm{LDH}$ relative to the null vector-transduced EPCs and nontransduced EPCs $(\mathrm{p}<0.05$; table 1$)$. These results confirmed that hTERT transduction of EPCs bolsters their NO, iNOS, and LDH secretory capacity in vitro.

Next, 120 healthy male rats were randomly segregated into four equally-sized groups: an HC group, an untreated AS group, a null EPC AS group, and an hTERT EPC AS group. The HC group was fed a normal diet for 3 months, while the three AS groups were fed a high-fat diet. Serum total cholesterol, LDL-C, triglyceride, and glucose levels were assayed in order to verify that all three AS groups showed statistically similar cholesterol and triglyceride levels prior to EPC injection. As a result, all three AS groups showed significantly higher serum total cholesterol, LDL-C, triglyceride, and glucose levels relative to the normally fed HC group ( $p<0.05$; table 2 ), while all three AS groups displayed statistically similar levels of total cholesterol, LDL-C, triglycerides, and glucose ( $\mathrm{p}>$ 0.05 ; table 2).

Then, the three EPC types were then injected into their respective AS rat groups in order to assess their effects in treating dietary-induced early AS. Two months after EPC injection, inverted fluorescent microscopy was applied to confirm EPC integration into the aortic tissue of recipient 


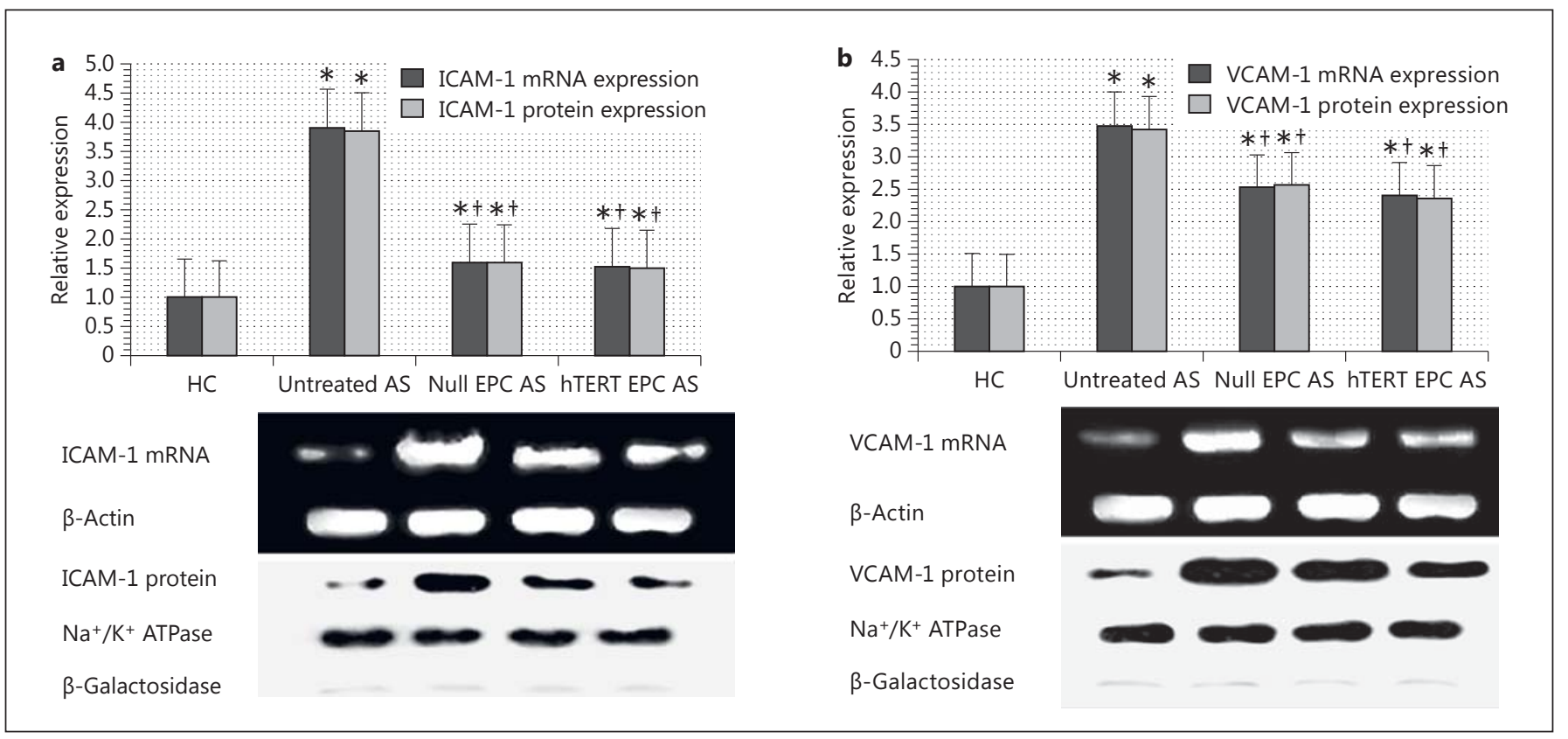

Fig. 3. Comparative expression of ICAM-1 and VCAM-1. The mRNA and surface protein expression of ICAM-1 (a) and VCAM1 (b) were assessed through RT-PCR and biotinylation, respectively. The untreated AS model showed significantly higher mRNA and surface protein expression of both ICAM-1 and VCAM-1 $(p<0.05)$. The injection of null vector-transduced EPCs and hTERT-transduced EPCs into the AS model resulted in a signifi- cant reduction in both ICAM-1 and VCAM-1 expression ( $\mathrm{p}<$ $0.05)$. However, there was no significant difference in either ICAM-1 or VCAM-1 expression between injection with null vector-transduced EPCs and injection with hTERT-transduced EPCs $(\mathrm{p}>0.05){ }^{*} \mathrm{p}<0.05$ relative to the $\mathrm{HC}$ group ${ }^{\dagger} \mathrm{p}<0.05$ relative to the untreated AS group.

the AS model (fig. 4). The untreated AS model showed significantly higher mRNA and surface protein expression of LFA-1 ( $p<0.05$; fig. 4a), Mac-1 ( $p<0.05$; fig. 4b), and CD44 ( $p<0.05$; fig. $4 \mathrm{c}$ ). Notably, the injection of null vector-transduced EPCs and hTERT-transduced EPCs into the AS model resulted in a significant reduction in LFA-1 ( $<<0.05$; fig. $4 \mathrm{a})$, Mac-1 ( $\mathrm{p}<0.05$; fig. $4 \mathrm{~b}$ ), and CD44 ( $\mathrm{p}<0.05$; fig. $4 \mathrm{c}$ ). There was a significant difference in Mac-1 mRNA expression ( $p<0.05$; fig. $4 \mathrm{~b}$ ) as well as CD44 mRNA and surface protein expression $(\mathrm{p}<0.05$; fig. 4c) between injection with null vector-transduced EPCs and injection with hTERT-transduced EPCs. However, there was no significant difference in LFA-1 expression ( $p>0.05$; fig. 4a) or Mac-1 surface protein expression ( $p>0.05$; fig. $4 b$ ) between injection with null vectortransduced EPCs and injection with hTERT-transduced EPCs.

Finally, we assessed mRNA and protein expression of the monocytic chemokine MCP-1, the apolipoprotein apoE, and the tonal regulator eNOS in the aortic tissue samples of the AS model (fig. 5). The untreated AS model showed significantly higher mRNA and surface protein 


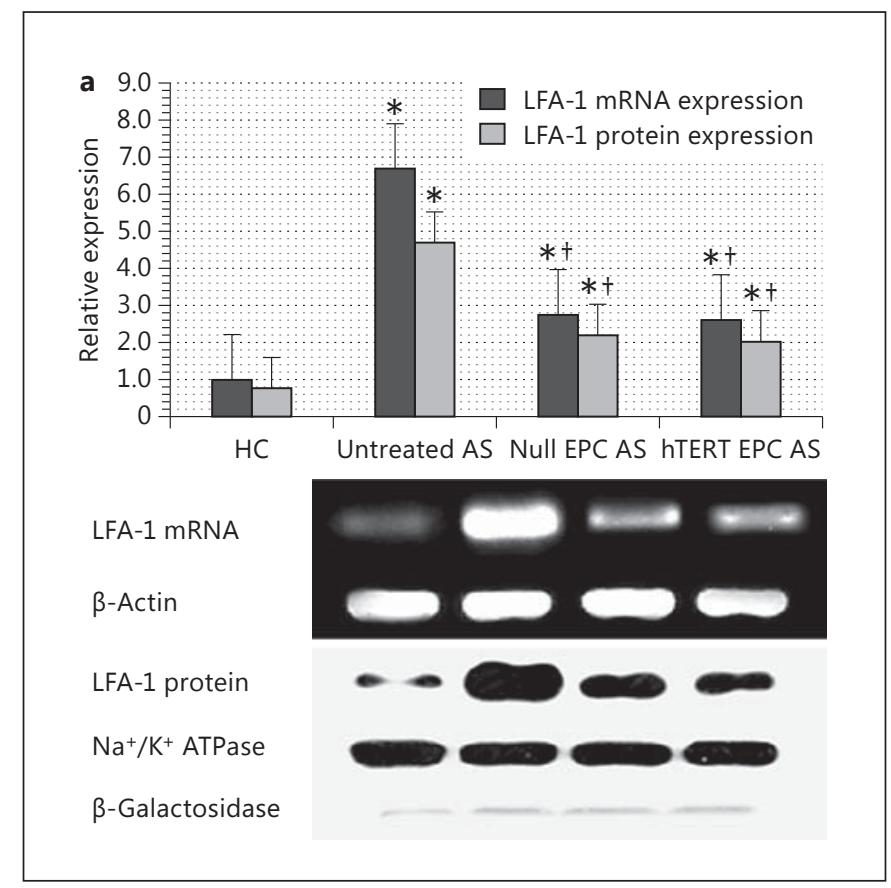

Fig. 4. Comparative expression of LFA-1, Mac-1, and CD44. The mRNA and surface protein expression of LFA-1 (a), Mac-1 (b), and CD44 (c) were assessed through RT-PCR and biotinylation, respectively. The untreated AS model showed significantly higher mRNA and surface protein expression of LFA-1, Mac-1, and CD44 $(\mathrm{p}<0.05)$. The injection of null vector-transduced EPCs and hTERT-transduced EPCs into the AS model resulted in a significant reduction in LFA-1, Mac-1, and CD44 expression ( $\mathrm{p}<0.05)$. There was a significant difference in Mac- 1 mRNA expression as well as CD44 mRNA and surface protein expression between injection with null vector-transduced EPCs and injection with hTERT-transduced EPCs $(\mathrm{p}<0.05)$. However, there was no significant difference in LFA-1 expression or Mac-1 surface protein expression between injection with null vector-transduced EPCs and injection with hTERT-transduced EPCs $(p>0.05) .{ }^{*} p<0.05$ relative to the $\mathrm{HC}$ group, ${ }^{\dagger} \mathrm{p}<0.05$ relative to the untreated AS group, ${ }^{\ddagger} \mathrm{p}<0.05$ relative to the null EPC AS group.

expression of MCP-1 ( $<<0.05$; fig. 5a) and apoE ( $<<0.05$; fig. $5 \mathrm{~b}$ ) but significantly lower expression of eNOS ( $<<$ 0.05; fig. 5c). The injection of null vector-transduced EPCs and hTERT-transduced EPCs into the AS model resulted in a significant reduction in both MCP-1 expression ( $\mathrm{p}<$ 0.05 ; fig. 5a) and apoE expression ( $\mathrm{p}<0.05$; fig. $5 \mathrm{~b})$ but significantly higher expression of eNOS ( $p<0.05$; fig. $5 c$ ).

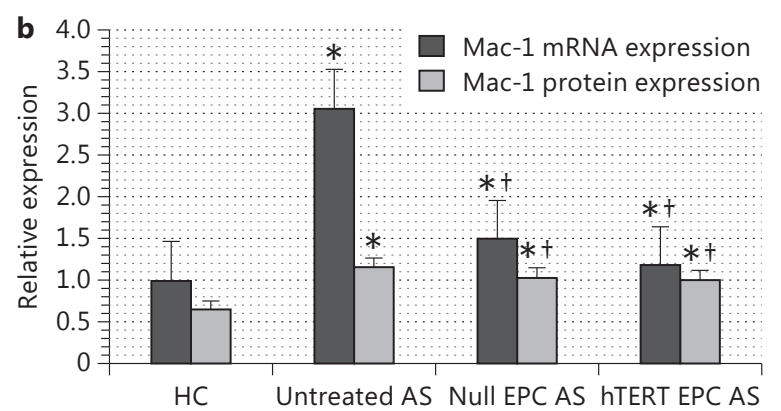

Mac-1 mRNA

$\beta$-Actin

Mac-1 protein

$\mathrm{Na}^{+} / \mathrm{K}^{+}$ATPase

$\beta$-Galactosidase
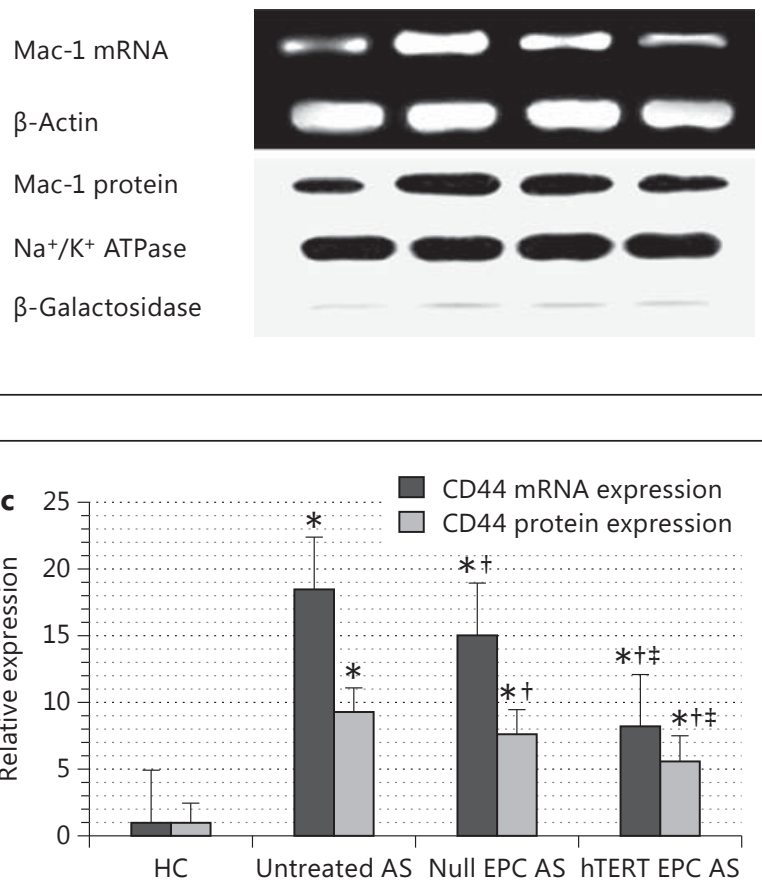

CD44 mRNA

$\beta$-Actin

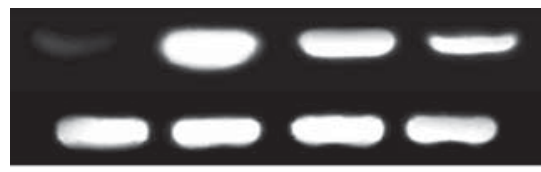

CD44 protein

$\mathrm{Na}^{+} / \mathrm{K}^{+}$ATPase

$\beta$-Galactosidase

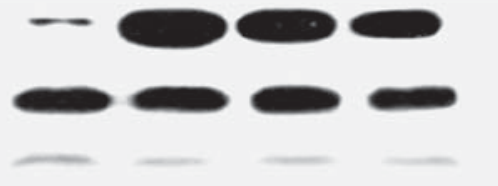

There was a significant difference in MCP-1 expression ( $\mathrm{p}<0.05$; fig. 5a) and eNOS expression ( $<<0.05$; fig. $5 \mathrm{c}$ ) between injection with null vector-transduced EPCs and injection with hTERT-transduced EPCs. However, there was no significant difference in apoE expression between injection with null vector-transduced EPCs and injection with hTERT-transduced EPCs ( $p>0.05$; fig. 5b). 


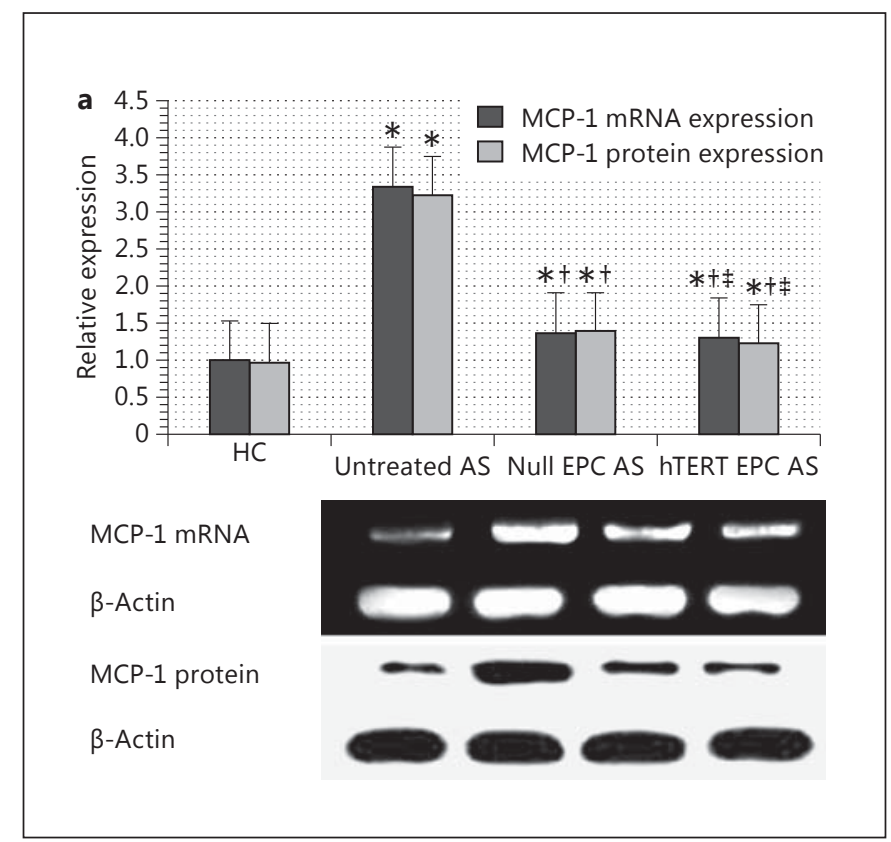

Fig. 5. Comparative expression of MCP-1, apoE, and eNOS. The mRNA and protein expression of MCP-1 (a), apoE (b), and eNOS (c) were assessed through RT-PCR and Western blotting, respectively. The untreated AS model showed significantly higher mRNA and surface protein expression of MCP-1 and apoE but significantly lower expression of eNOS $(p<0.05)$. The injection of null vector-transduced EPCs and hTERT-transduced EPCs into the AS model resulted in a significant reduction in both MCP-1 and apoE expression but significantly higher expression of eNOS ( $\mathrm{p}<0.05)$. There was a significant difference in MCP-1 and eNOS expression between injection with null vector-transduced EPCs and injection with hTERT-transduced EPCs $(\mathrm{p}<0.05)$. However, there was no significant difference in apoE expression between injection with null vector-transduced EPCs and injection with hTERT-transduced EPCs $(\mathrm{p}>0.05) .{ }^{*} \mathrm{p}<0.05$ relative to the $\mathrm{HC}$ group, ${ }^{\dagger} \mathrm{p}<$ 0.05 relative to the untreated AS group, ${ }^{\ddagger} \mathrm{p}<0.05$ relative to the null EPC AS group.

\section{Discussion}

In this study we hypothesized that transduction of the hTERT gene would enhance EPC function in treating dietary-induced early AS. In vitro, we found that exogenous hTERT transduction of EPCs resulted in a significantly superior proliferative capacity as well as a significantly higher NO, iNOS, and LDH secretory capacity. From the aortic tissue samples resected from the AS model, we
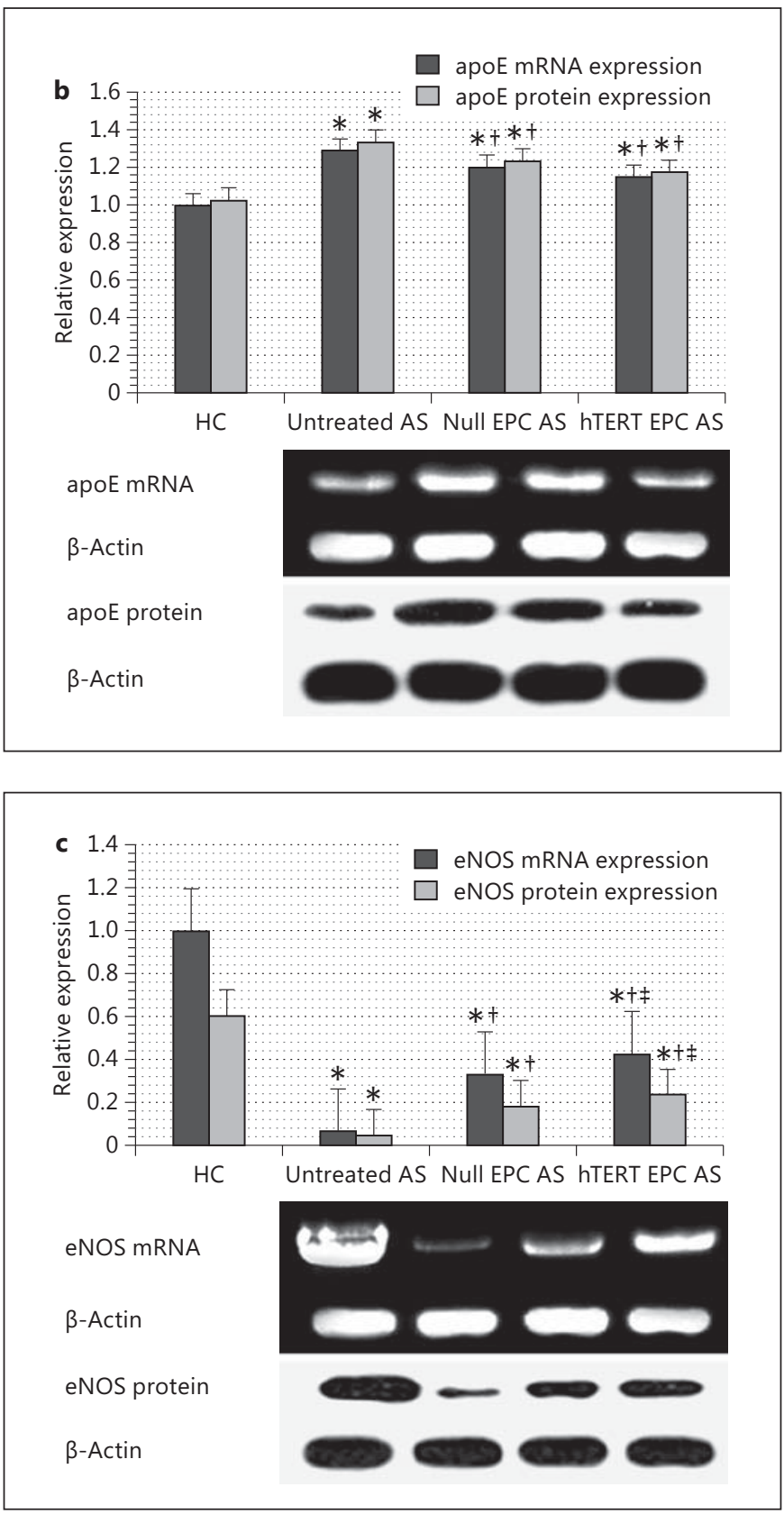

found that injection with exogenous hTERT-transduced EPCs produced significant reductions in CD44 and MCP1 expression as well as a significant increase in eNOS expression relative to injection with null vector-transduced EPCs.

In the process of dietary-induced early AS, circulating atherogenic lipoproteins diffuse into and accumulate within the extracellular intimal space [11]. Activated monocytes/macrophages then migrate into the ex- 
tracellular intimal space and phagocytize these lipoproteins, which transforms them into lipid-laden foam cells [11]. The resulting foam cell accumulation produces fatty streaks, which have been observed in the major arteries of children and adolescents [11]. Next, vascular smooth muscle cells secrete extracellular matrix proteins, which further compounds the accumulation of atherogenic lipoproteins in the early atherosclerotic lesion. Finally, leukocytes - such as monocytes and $\mathrm{T}$ cells - are recruited into the early atherosclerotic lesion, which produces a localized area of chronic inflammation that eventually develops into a mature atherosclerotic plaque [11].

As the endothelium forms the luminal surface of atherosclerotic lesions, endothelial cells play a key role in recruiting leukocytes to early atherosclerotic lesions [11]. As described above, the resulting infiltration of leukocytes into early atherosclerotic lesions plays an important role in the further progression of AS [11]. Specifically, the recruitment of leukocytes by endothelial cells is dependent upon the surface expression of certain key adhesion molecules, such as ICAM-1, VCAM-1, and CD44 [12]. With specific respect to CD44, several lines of evidence suggest that increased CD44 expression plays a role in atherogenesis. For example, the expression of CD44 has been shown to be upregulated in macrophage-laden atherosclerotic lesions relative to simple fibrous lesions [13], and several CD44 ligands have been shown to be upregulated in the extracellular matrix of atherosclerotic lesions [14]. Moreover, CD44 expression has been shown to be upregulated in the vessel walls of apoE-deficient mice during early AS $[6,15]$, while CD44-null mice display reduced activated monocyte/macrophage recruitment to atherosclerotic lesions as well as reductions in atherosclerotic lesion size [16]. Here, we found that injection of exogenous hTERT-transduced EPCs produced significant reductions in CD44 expression in aortic tissue samples from the AS model. This finding suggests that hTERTtransduced EPCs may be an effective tool in reducing activated monocyte/macrophage recruitment to early atherosclerotic lesions.

As discussed above, during early AS, activated monocytes/macrophages migrate into the extracellular intimal space, where they phagocytose deposited atherogenic lipoproteins [11]. Several lines of evidence show that these activated monocytes/macrophages are chemotactically directed to the early lesion via the secretion of MCP-1 by endothelial cells and vascular smooth muscle cells [17]. For example, MCP-1 expression is significantly upregulated in intimal macrophages in early atherosclerotic le- sions, with MCP-1 expression being highly correlated with macrophagic accumulation [18, 19]. Moreover, MCP-1-deficient mice on a high cholesterol diet display significantly lower lipid deposition and lower macrophage counts in their aortic walls [20]. Here, we found that injection of exogenous hTERT-transduced EPCs produced significant reductions in MCP-1 expression in aortic tissue samples from the AS model. This finding suggests that hTERT-transduced EPCs may be an effective tool in reducing activated monocyte/macrophage recruitment to early atherosclerotic lesions.

The disturbance of laminar blood flow in certain nonlinear vascular segments is thought to facilitate atherosclerotic progression through promoting shear stress-induced endothelial dysfunction $[21,22]$. In particular, Heo et al. [23] demonstrated that disturbed laminar flow downregulates endothelial cell production of the enzyme eNOS. As this eNOS downregulation action reduces NO synthesis [24], this process can promote atherosclerotic progression through dysregulating adhesion molecule expression, platelet activation, and vascular smooth muscle activity [25]. Here, we found that injection of exogenous hTERT-transduced EPCs produced significant increases in eNOS expression in aortic tissue samples from the AS model. This finding suggests that hTERT-transduced EPCs may be an effective tool in countering atherosclerotic progression.

There are several limitations to this study. First, this investigation was performed in a rat model of dietaryinduced early AS. Therefore, our findings may not directly translate to dietary-induced early AS in humans. Second, only aortic tissue samples extracted from the AS model were probed for early atherosclerotic marker expression. Thus, there is insufficient evidence to draw concrete conclusions across all arteries. Third, although we endeavored to assess expressional changes in the key markers of early AS, there are other early atherosclerotic markers, such as cathepsin B and COX-2, which we failed to examine here. Fourth, the use of viral vectors can create insertional mutations in EPCs that may disrupt the long-term functioning and differentiation in vivo [26]. Therefore, further studies are needed to assess the long-term effects of hTERT transduction upon EPCs in vivo.

In conclusion, the injection of exogenous hTERTtransduced EPCs appears to be superior to that of null vector-transduced EPCs in treating early AS in a high-fat dietary rat model of early AS. These findings suggest that an hTERT-transduced human EPC line may be a useful tool in treating dietary-induced early AS. 


\section{Acknowledgments}

This study was supported by the Natural Science Foundation Project of Chongqing (grant No. cstc2012jjA10088), the National Science Foundation of China (grant No. 81402474), and the Outstanding Young Talent Project of the Second Affiliated Hospital of Chongqing Medical University (grant No. 2014-43). The funders had no role in study design, data collection and analysis, decision to publish, or preparation of the manuscript.

\section{Conflict of Interest}

The authors have no conflicts of interest to disclose.

\section{References}

1 Matsuzawa Y, Guddeti RR, Kwon T-G, Lerman LO, Lerman A: Secondary prevention strategy of cardiovascular disease using endothelial function testing. Circ J 2015;79:685694.

2 Choi B-J, Matsuo Y, Aoki T, Kwon T-G, Prasad A, Gulati R, Lennon RJ, Lerman LO, Lerman A: Coronary endothelial dysfunction is associated with inflammation and vasa vasorum proliferation in patients with early atherosclerosis. Arterioscler Thromb Vasc Biol 2014;34:2473-2477.

3 Tousoulis D, Psaltopoulou T, Androulakis E, Papageorgiou N, Papaioannou S, Oikonomou E, Synetos A, Stefanadis C: Oxidative stress and early atherosclerosis: novel antioxidant treatment. Cardiovasc Drugs Ther 2015;29: $75-88$.

4 Dimmeler S, Zeiher AM: Vascular repair by circulating endothelial progenitor cells: the missing link in atherosclerosis? J Mol Med 2004;82:671-677.

5 Li S-H, Wang D-D, Xu Y-J, Ma G-D, Li X-Y, Liang W-J: Exogenous hTERT gene transfected endothelial progenitor cells from bone marrow promoted angiogenesis in ischemic myocardium of rats. Int J Clin Exp Med 2015; $8: 14447$.

$6 \mathrm{Ma}$ Y, Malbon CC, Williams DL, Thorngate FE: Altered gene expression in early atherosclerosis is blocked by low level apolipoprotein e. PLoS One 2008;3:e2503.

7 Wang F, Xue J, Wang D, Wang X, Lu S, Tan $\mathrm{M}$ : Treatment of atherosclerosis by transplantation of bone endothelial progenitor cells over-expressed paraoxonase- 1 gene by recombinant adeno-associated virus in rat. Biol Pharm Bull 2010;33:1806-1813.

8 Kalka C, Masuda H, Takahashi T, Kalka-Moll WM, Silver M, Kearney M, Li T, Isner JM, Asahara T: Transplantation of ex vivo expanded endothelial progenitor cells for therapeutic neovascularization. Proc Natl Acad Sci USA 2000;97:3422-3427.
9 Yuan JS, Reed A, Chen F, Stewart CN: Statisti$\mathrm{cal}$ analysis of real-time PCR data. BMC Bioinformatics 2006;7:1.

10 Lei H, Romeo G, Kazlauskas A: Heat shock protein $90 a$-dependent translocation of annexin II to the surface of endothelial cells modulates plasmin activity in the diabetic rat aorta. Circ Res 2004;94:902-909.

11 Rader DJ, Daugherty A: Translating molecular discoveries into new therapies for atherosclerosis. Nature 2008;451:904-913.

12 Butcher MJ, Galkina E: Mechanisms of Leukocyte Recruitment into the Aorta during Atherosclerosis. Rijeka, InTech, 2012.

13 Krettek A, Sjoberg S: CD44 - a new cardiovascular drug target or merely an innocent bystander? Cardiovasc Haematol Disord Drug Targets 2009;9:293-302.

14 Xiao H-B, Lu X-Y, Sun Z-L, Zhang H-B: Kaempferol regulates OPN-CD44 pathway to inhibit the atherogenesis of apolipoprotein $\mathrm{E}$ deficient mice. Toxicol Appl Pharmacol 2011; 257:405-411.

15 Zhao L, Hall JA, Levenkova N, Lee E, Middleton MK, Zukas AM, Rader DJ, Rux JJ, Puré E: $\mathrm{CD} 44$ regulates vascular gene expression in a proatherogenic environment. Arterioscler Thromb Vasc Biol 2007;27:886-892.

16 Cuff CA, Kothapalli D, Azonobi I, Chun S, Zhang Y, Belkin R, Yeh C, Secreto A, Assoian RK, Rader DJ: The adhesion receptor CD44 promotes atherosclerosis by mediating inflammatory cell recruitment and vascular cell activation. J Clin Invest 2001;108:1031.

17 Cushing SD, Berliner JA, Valente AJ, Territo MC, Navab M, Parhami F, Gerrity R, Schwartz CJ, Fogelman AM: Minimally modified low density lipoprotein induces monocyte chemotactic protein 1 in human endothelial cells and smooth muscle cells. Proc Natl Acad Sci USA 1990;87:5134-5138.
18 Kowala MC, Recce R, Beyer S, Gu C, Valentine $\mathrm{M}$ : Characterization of atherosclerosis in LDL receptor knockout mice: macrophage accumulation correlates with rapid and sustained expression of aortic MCP-1/JE. Atherosclerosis 2000;149:323-330.

19 Nakashima Y, Fujii H, Sumiyoshi S, Wight TN, Sueishi K: Early human atherosclerosis accumulation of lipid and proteoglycans in intimal thickenings followed by macrophage infiltration. Arterioscler Thromb Vasc Biol 2007;27:1159-1165.

20 Gu L, Okada Y, Clinton SK, Gerard C, Sukhova GK, Libby P, Rollins BJ: Absence of monocyte chemoattractant protein- 1 reduces atherosclerosis in low density lipoprotein receptor-deficient mice. Mol Cell 1998;2:275281.

21 Cybulsky MI, Marsden PA: Effect of disturbed blood flow on endothelial cell gene expression a role for changes in RNA processing. Arterioscler Thromb Vasc Biol 2014;34: 1806-1808.

22 Heo K-S, Fujiwara K, Abe J: Shear stress and atherosclerosis. Mol Cells 2014;37:435.

23 Heo K-S, Berk B, Abe J: Disturbed flow-induced endothelial proatherogenic signaling via regulating post-translational modifications and epigenetic events. Antioxid Redox Signal 2016, Epub ahead of print.

24 Shaul PW, Smart EJ, Robinson LJ, German Z, Yuhanna IS, Ying Y, Anderson RG, Michel T: Acylation targets endothelial nitric-oxide synthase to plasmalemmal caveolae. J Biol Chem 1996;271:6518-6522.

25 Oemar BS, Tschudi MR, Godoy N, Brovkovich V, Malinski T, Lüscher TF: Reduced endothelial nitric oxide synthase expression and production in human atherosclerosis. Circulation 1998;97:2494-2498.

$26 \mathrm{Yu}$ J, Hu K, Smuga-Otto K, Tian S, Stewart R, Slukvin II, Thomson JA: Human induced pluripotent stem cells free of vector and transgene sequences. Science 2009;324:797-801. 